\title{
Combinatorial mechanical gradation and growth factor biopatterning strategy for spatially controlled bone-tendon-like cell differentiation and tissue formation
}

Dan Wang ${ }^{1,2,3,4,5}$, Dai Fei Elmer Ker $\mathbb{B}^{1,2,3,4,5}$, Ka Wai Ng${ }^{2}, K^{2} \mathrm{Li}^{2,3}$, Burhan Gharaibeh ${ }^{6}$, Marc Safran ${ }^{1}$, Emilie Cheung ${ }^{1}$, Phil Campbell ${ }^{7,8}$, Lee Weiss ${ }^{9,8}$ and Yunzhi Peter Yang (10,10,11

\begin{abstract}
Engineering scaffolds to augment the repair of hard-to-soft multitissue musculoskeletal tissue units, such as bonetendon, to simultaneously support tissue healing and functional movement has had limited success. Overcoming this challenge will require not only precise spatial control of bone- and tendon-like biomechanical properties, but also consideration of the resultant biomechanical cues, as well as the embedded biochemical cues imparted by these scaffolds. Here, we report on the effects of a spatially engineered combination of stiffness and growth factor (GF) cues to control bone-tendon-like differentiation in vitro and tissue formation in vivo. This was achieved using mechanically graded, bone- and tendon-like QHM polyurethane (QHM: Q: Quadrol; H: hexamethylene diisocyanate; M: methacrylic anhydride) scaffolds selectively biopatterned with osteogenic bone morphogenetic protein-2 (BMP-2) and tenogenic fibroblast growth factor-2 (FGF-2). First, material characterization, including porosity, surface roughness, contact angle, and microindentation measurements, was performed. Second, in vitro studies demonstrated that increased material stiffness promoted GF-mediated osteoblast differentiation and reduced tenocyte differentiation. Sustained GF exposure masked this stiffness effect. Third, in vivo studies involving subcutaneous implantation of mechanically graded and biochemically patterned QHM scaffolds (composed of these bone- and tendon-promoting GFs biopatterned on biphasic bone and tendon biomechanically mimicking regions) in mice demonstrated spatial control of bone- and tendon-like tissue formation. Altogether, these data provide new insights for future engineering of scaffolds to augment hard-to-soft multitissue repair.
\end{abstract}

\section{Introduction}

Musculoskeletal tissue units are composed of different tissues and cell types that are organized in a functionally graded, spatially specific manner to mediate musculoskeletal tissue attachment and joint movement. The

\footnotetext{
Correspondence: Dai Fei Elmer Ker (elmerker@cuhk.edu.hk) or Yunzhi Peter Yang (ypyang@stanford.edu)

'Department of Orthopaedic Surgery, Stanford University, 240 Pasteur Drive, Stanford, CA 94304, USA

${ }^{2}$ Institute for Tissue Engineering and Regenerative Medicine, The Chinese University of Hong Kong, Shatin, Hong Kong SAR

Full list of author information is available at the end of the article

These authors contributed equally: Dan Wang, Dai Fei Elmer Ker
}

bone-tendon interfaces of rotator cuff tissues are typical examples of hard-to-soft tissue units that often become injured $^{1}$. Repairing such multitissue units is challenging, as subsequent tissue healing is typically insufficient for recapitulating resident musculoskeletal cells, as well as the native tissue architecture and structure. In severe cases, unstable mechanical repair and inadequate biological healing lead to fibrovascular scar formation, resulting in biomechanically inferior tissue that is prone to retearing ${ }^{2}$. Therefore, tissue engineering strategies need to aim for biomimicry, simultaneously patterning mechanical and

\section{(c) The Author(s) 2021}

(c) (i) Open Access This article is licensed under a Creative Commons Attribution 4.0 International License, which permits use, sharing, adaptation, distribution and reproduction c. in any medium or format, as long as you give appropriate credit to the original author(s) and the source, provide a link to the Creative Commons license, and indicate if changes were made. The images or other third party material in this article are included in the article's Creative Commons license, unless indicated otherwise in a credit line to the material. If material is not included in the article's Creative Commons license and your intended use is not permitted by statutory regulation or exceeds the permitted use, you will need to obtain permission directly from the copyright holder. To view a copy of this license, visit http://creativecommons.org/licenses/by/4.0/. 
biochemical cues onto scaffolds to augment the repair site in a spatially precise manner.

For the repair of hard-to-soft multitissue units, there have been numerous investigations and advancements in the development of biomechanically or biochemically graded scaffolds in recent years. These include but are not limited to utilizing various biomaterials (synthetic, natural or combinatorial) fabricated via either multiphasic- or gradient-based approaches. For instance, multiphasic scaffolds can be engineered by joining different materials together either by sintering ${ }^{3}$ or a combination of sacrificial pore casting, layering, and laser machining ${ }^{4}$. Alternatively, gradient-based scaffolds can be engineered by establishing (1) porosity gradients through a combination of three-dimensional printing and controlled freezedrying$^{5}$; (2) mineral gradients via gel casting, layering, and controlled soaking in a mineral solution ${ }^{6,7}$; (3) mineralizing (osteoblast) cell gradients via coated retroviral gene transfer of an osteoblast-associated transcription factor (Runt-related transcription factor-2; runx2) ${ }^{8}$; or (4) biomechanical gradients achieved via photocrosslinking chemistry ${ }^{9}$. Despite considerable progress, successful reports of multitissue healing using such graded scaffolds in vivo have been limited. This is primarily due to the challenging dual requirements of achieving mechanical properties that closely approximate bonetendon tissues for sustaining shoulder loading and spatial patterning of multiple musculoskeletal phenotypes, including osteoblasts and tenocytes for repopulating tissue-resident cells $^{10-12}$. Crucially, linking these two important criteria is the biomechanical effect(s) that a scaffold exerts on infiltrating or preseeded cells. While prior studies have already investigated the effects of material stiffness on cell differentiation, they were largely based on microscale measurements ${ }^{4,13}$. The ability of a scaffold's macroscale mechanical properties to approximate physiological bone-to-tendon values, i.e., $0.2-29.0 \mathrm{GPa}$ tensile modulus ${ }^{1,14}$, for multitissue patterning is largely unexplored, especially in conjunction with scaffold bioinductive cue(s). Thus, an improved understanding of this effect will allow appropriate scaffold biomechanical cues to be engineered for promoting both biomechanical function and multitissue healing.

To address this, our study's focus was to engineer mechanically graded, bone- and tendon-like scaffolds combined with bioprinted growth factors (GFs) to investigate musculoskeletal cell differentiation in response to predesigned biomechanical and biochemical patterns. First, to achieve spatially controlled scaffold biomechanical properties, we utilized our previously developed UVcrosslinkable polyurethane (QHM polymers), which are formulated with Quadrol $(\mathrm{Q})$, hexamethylene diisocyanate $(\mathrm{H})$, and methacrylic anhydride $(\mathrm{M})^{9}$. QHM polymers exhibited phototunable bone- and tendon-like mechanical properties (tensile strength/yield: $12-74 \mathrm{MPa}$ and tensile modulus: $0.6-2.7 \mathrm{GPa}$ ), reduced stress concentrations via gradual stiffness gradients (achieved via selective photomasking), and showed capacity for longterm scaffold integrity (i.e., biocompatibility, slow degradation, and excellent suture retention) ${ }^{9}$. Here, additional material characterization, such as porosity, surface roughness, contact angle, and microindentation measurements, was performed. Second, we examined the combined effects of biomechanical cues (presented by the mechanical properties of the biphasic QHM polyurethane) and bone- and tendon-promoting GFs (either in soluble/liquid-phase form or biopatterned/solid-phase form) on osteogenic and tenogenic differentiation. Specifically, to achieve spatial control and immobilization of GF cues, we fibrin coated and biopatterned the bone-like (300 s UV) and tendon-like (0 s UV) regions of the QHM polymer with osteoinductive bone morphogenetic protein-2 (BMP-2) ${ }^{15-19}$ and tenogenic fibroblastic growth factor-2 (FGF-2) ${ }^{16,17}$, respectively. This was achieved by using our established biopatterning methodology whereby GFs are selectively printed on and absorbed into extracellular matrix (ECM) materials, where they become immobilized via native binding affinities ${ }^{15-18,20,21}$. These mechanically graded and biochemically patterned scaffolds were studied both in vitro using $\mathrm{C} 2 \mathrm{C} 12$ and C3H10T1/2 as mouse myoblast and mouse mesenchymal fibroblast cell lines, respectively, and in vivo using a mouse subcutaneous implantation model.

\section{Materials and methods \\ Material fabrication}

QHM polyurethane was fabricated using a solventand catalyst-free approach from N,N,N',N'-tetrakis (2hydroxy-propyl) ethylenediamine (Quadrol or Q), hexamethylene diisocyanate/1,6-diisocyanatohexane (H) and methacrylic anhydride (M) (Sigma Aldrich, USA), as previously described ${ }^{9}$. Briefly, $\mathrm{Q}, \mathrm{H}$, and $\mathrm{M}$ were reacted at a molar ratio of 1:1.5:0.5 and exposed to 0,90 , 180, and 300 s UV light (OmniCure Series 2000 UV system, Excelitas Technologies, USA) ${ }^{9}$ as depicted in Fig. 1. For more details, please refer to the Supplementary Information.

\section{Porosity measurements}

Mercury intrusion porosity measurements were performed on QHM polymer samples (circular discs $\sim 1 \mathrm{~cm}$ in diameter) by Micromeritics analytical services (Micromeritics, Norcross, GA).

\section{Surface profile measurements}

Surface profiling was performed using a Veeco Dektak 150 Profilometer (Veeca Instruments Inc., Plainview, NY) equipped with a $25 \mu \mathrm{m}$ diameter stylus tip. Line profiles 


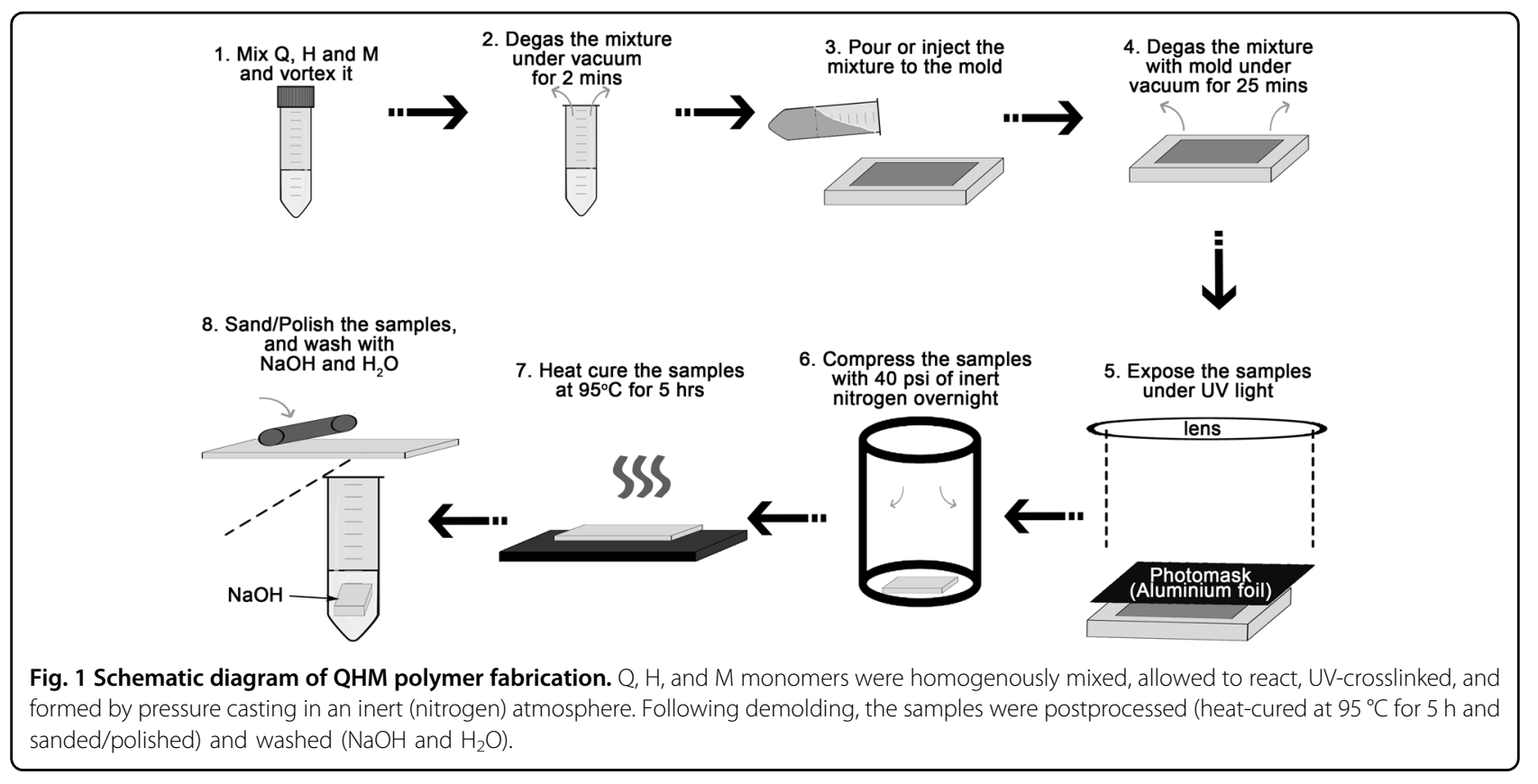

(1 $\mathrm{mm}$ ) were obtained at a resolution of $28 \mathrm{~nm}$ per QHM polymer sample (circular discs $\sim 1 \mathrm{~cm}$ in diameter).

\section{Contact angle measurements}

Contact angle measurements were performed by pipetting $1 \mu \mathrm{L}$ of deionized water onto QHM polymer samples (circular discs $\sim 1 \mathrm{~cm}$ in diameter) followed by imaging using a Canon PowerShot S95 camera at room temperature. Contact angle measurements were made using ImageJ software (http://imagej.nih.gov/ij/, National Institutes of Health, Bethesda, MD).

\section{Microindentation tests}

Microindentation tests were performed using an FM series microhardness testing machine (Future-Tech Corp, Japan) with an indenter of standardized size and maximum diagonal length of $1.4 \mathrm{~mm}$ according to ISO 6507. The indenter compressed the region of interest (ROI) with a force of $20 \mathrm{mN}$, dwelling for $10 \mathrm{~s}$. Following this, the elastic modulus of the QHM polymer samples was computed during an unloading hold of $5 \mathrm{~s}$.

\section{Fibrin coating}

QHM polymer samples were fibrin coated as previously described $^{9,}$ and the thickness of the fibrin films was previously estimated by transmission electron microscopy to be $\sim 20 \mathrm{~nm}^{17,20}$. For more details, please refer to the Supplementary Information.

\section{GF biopatterning}

Recombinant human BMP-2 (Medtronic, USA) and FGF-2 (PeproTech, USA) patterning was performed using either our custom-built inkjet-based bioprinter as previously described ${ }^{15-17}$ or manually with a micropipette. The bioprinter was used for those experiments requiring high-resolution patterns, while pipetting was used when lower resolutions were sufficient to demonstrate biopatterning efficacy. The trade-off is that bioprinting with the current printer is time-consuming since it incorporates a printhead with a single $30 \mu \mathrm{m}$ diameter nozzle per GF, whereas micropipetting can be rapidly performed. As described in our prior work ${ }^{15-17}$, GF was loaded into the printhead and biopatterned onto fibrin-coated QHM polymer samples. The concentration of biopatterned GFs can be modulated by overprinting, which was achieved by varying the number of times a GF was deposited in the same $(x, y)$ location. Alternatively, fibrin-coated QHM polymer samples were manually biopatterned by using a micropipette to deposit $0.5-3 \mu \mathrm{L}$ of GF. To minimize fibrin degradation, $1 \mu \mathrm{g} / \mathrm{mL}$ aprotinin was directly added to culture medium as described, or fibrin-coated QHM polymers were incubated with $1 \mu \mathrm{g} / \mathrm{mL}$ aprotinin overnight after fibrin coating, allowed to dry and stored at $4{ }^{\circ} \mathrm{C}$ prior to GF printing for in vivo studies. For more details, please refer to the Supplementary Information.

\section{GF immobilization studies}

The surface concentration of GF present on fibrincoated QHM (circular discs $\sim 1 \mathrm{~cm}$ in diameter) was measured using fluorescently labeled GFs. BMP-2 and FGF-2 were fluorescently labeled with an Amine-Reactive DyLight 650 NHS-ester Conjugation kit (Pierce Biotechnology, USA), and unbound dye was removed using dye removal columns (Pierce Biotechnology, USA). The 
degree of labeling was monitored using a Biophotometer spectrophotometer (Eppendorf, Germany). To perform desorption measurements, $0.5 \mu \mathrm{L}$ of GFs at various concentrations were handprinted onto fibrin-coated QHM polymer samples, allowed to dry for $1 \mathrm{~h}$ at $37^{\circ} \mathrm{C}$ and fluorescently imaged prior to washing. Subsequently, samples were incubated in PBS for $5 \mathrm{~min}$ and fluorescently imaged using an inverted Zeiss AxioObserver Z1 microscope. A total of three rounds of sample washing and fluorescence imaging were performed. Fluorescence signals were quantified by calculating the average pixel intensity in Adobe Photoshop 7.0 (Adobe Systems, USA), and fluorescence intensity was normalized to their corresponding unwashed controls. For more details, please refer to the Supplementary Information.

\section{Cell culture}

Multipotent mouse $\mathrm{C} 2 \mathrm{C} 12$ myoblasts and mouse C3H10T1/2 fibroblasts (ATTC, USA) were used as surrogate models of muscle-derived and mesenchymal stem cells. Hoechst 33342 staining (Anaspec, USA) determined that cell cultures were free of mycoplasma contamination. C2C12 or C3H10T1/2 cells were cultured at a density of $7.47 \times 10^{4}$ cells per $\mathrm{cm}^{2}$ in growth medium (Dulbecco's modified Eagle medium, 10\% fetal bovine serum, 1\% penicillin-streptomycin) on QHM polymers (circular discs $\sim 1 \mathrm{~cm}$ in diameter) with different material stiffnesses in 24well plates for designated times. BMP-2 (100 or $200 \mathrm{ng} / \mathrm{mL}$ for liquid-phase studies and $100 \mu \mathrm{g} / \mathrm{mL}$ for biopatterning studies) or FGF-2 (50 or $100 \mathrm{ng} / \mathrm{mL}$ for liquid-phase studies and $100 \mu \mathrm{g} / \mathrm{mL}$ for biopatterning studies) was added directly into the cell culture medium or biopatterned on fibrin-coated QHM polymer samples with $1 \mu \mathrm{g} / \mathrm{mL}$ aprotinin (Sigma Aldrich, USA). For studies on the effect of tensile modulus (material stiffness) on osteoblast differentiation (using uniform QHM polymer samples), C2C12 cells were seeded in growth medium overnight (Day 0), which was changed to growth medium containing $100 \mathrm{ng} /$ $\mathrm{mL}$ BMP-2 and 0.3\% dimethyl sulfoxide (DMSO) or growth medium with $100 \mathrm{ng} / \mathrm{mL}$ BMP-2 and $25 \mu \mathrm{M}$ blebbistatin (Selleck Chemicals, USA) the following day. For studies on the effect of tensile modulus on osteoblast-mediated mineralization, $\mathrm{C} 2 \mathrm{C} 12$ cells were seeded in growth medium overnight (Day 0), which was changed to growth medium and $1 \mu \mathrm{g} / \mathrm{mL}$ aprotinin (control media) or growth medium containing $10 \mathrm{mM} \beta$-glycerophosphate, $50 \mu \mathrm{g} / \mathrm{mL}$ ascorbic acid, $100 \mathrm{ng} / \mathrm{mL}$ BMP-2, and $1 \mu \mathrm{g} / \mathrm{mL}$ aprotinin (osteogenic medium). For more details, please refer to the Supplementary Information.

\section{ALP, von Kossa, and immunofluorescence staining}

At designated time points, cells were fixed for $1 \mathrm{~min}$ in $3.7 \%$ formaldehyde, and samples were stained. Alkaline phosphatase (ALP; Sigma Aldrich, USA), von Kossa
(American MasterTech Scientific, USA) and immunofluorescence staining were performed as previously described according to the manufacturer's instructions ${ }^{16,17}$. For immunofluorescence staining, the primary antibodies included rabbit anti-scleraxis (anti-SCX), mouse anti-tenascin $\mathrm{C}$ (anti-TNC) and rabbit antitenomodulin (anti-TNMD) (Abcam, USA). Images were captured using a Zeiss Axiovert $200 \mathrm{M}$ microscope (Carl Zeiss Microimaging, USA). For more details, please refer to the Supplementary Information.

\section{Mouse subcutaneous dorsal model}

Mouse subcutaneous dorsal surgeries were performed in accordance with the guidelines established by Stanford University's Administrative Panel on Laboratory Animal Care. Wild-type C57BL-6J mice (Charles River Laboratories, USA) between 8 and 12 weeks of age (20-30 g) were used in this study. Scaffolds (square specimens $6 \times$ $6 \times 1 \mathrm{~mm}$ ) comprised $0 \mathrm{~s}$ UV and $300 \mathrm{~s}$ UV QHM polymer regions (each measuring $3 \times 3 \times 1 \mathrm{~mm}$ ) biopatterned with (1) no GF, (2) $3 \mu \mathrm{g}$ of FGF-2 on the tendon-like $0 \mathrm{~s}$ UV QHM polymer region and $3 \mu \mathrm{g}$ of BMP-2 on the bone-like 300 s UV QHM polymer region. Under sterile conditions, a $2.5 \mathrm{~cm}$ skin incision was made dorsally, and the scaffolds were positioned such that the GF-biopatterned surfaces were in physical contact with the skin. Once properly positioned, the scaffolds were sutured to the skin subcutaneously, and at 2 weeks postsurgery, samples were harvested. For more details, please refer to the Supplementary Information.

\section{Histological analyses}

For the mouse subcutaneous dorsal model, harvested samples were fixed in $4 \%$ paraformaldehyde, and samples were subsequently processed for immunofluorescence (anti-SCX) or histological staining. Histological staining for hematoxylin and eosin (H\&E), tartrate-resistant acid phosphatase (TRAP) and Lillie Modification of Mason's Trichrome (Tri) were performed according to the manufacturer's instructions (Electron Microscopy Sciences, USA). Images of samples were acquired using either an inverted Zeiss AxioObserver Z1 microscope equipped with an AxioCam ICC color camera or an upright Zeiss Axioimager polarized microscope (Pol; Carl Zeiss Microimaging, USA) equipped with a Zeiss Axiocam 506 color camera (Carl Zeiss Microimaging, USA). For more details, please refer to the Supplementary Information.

\section{Statistical analysis}

Sample sizes for each experiment are indicated in each figure legend and were estimated to detect a group mean difference of $50 \% \pm 1$ to 2 standard deviations with a power $(1-\beta)$ of 0.8 and $\alpha=0.05$. The Shapiro-Wilk test and the Levene test were used to determine whether the 
data were normally distributed and had equal variances among groups, respectively. For multiple comparisons, one-way or two-way analysis of variance (ANOVA) was performed using SYSTAT 12 (Systat Software., USA) or SPSS Statistics 23 (IBM., USA) software. For one-way ANOVA, pairwise comparisons were performed using Tukey's honestly significant difference post hoc test (on data that satisfied normality or equal variance assumptions) or Games-Howell post hoc test (on data that did not satisfy both normality and equal variance assumptions to enable improved control of Type I error and greater power under conditions of nonnormality and heterogeneity of variance $)^{22}$. For two-way ANOVA, simpleeffects analysis was performed when a significant interaction was found. A $p$-value $\leq 0.05$ was considered statistically significant. For more details, please refer to the Supplementary Information.

\section{Results}

Effect of polymer fabrication (UV exposure) and postprocessing (sanding) on uniform QHM polymer (nongraded) porosity, roughness, and hydrophobicity (contact angle)

Prior to in vitro studies, it is crucial to determine the effect of polymer fabrication and postprocessing steps on QHM polymer properties, such as porosity, pore size, surface roughness, and hydrophobicity (contact angle), as these biomaterial attributes can regulate cell behavior and matrix remodeling ${ }^{23-25}$. Polymer fabrication procedures such as UV light-induced crosslinking showed minor differences in QHM polymer porosity, with $0 \mathrm{~s} \mathrm{UV}$ and 300 s UV QHM polymers exhibiting 10 and 7\% porosity, respectively (Fig. 2A, B). In general, increased UV exposure time resulted in reduced porosity, but pore sizes remained similar at $\sim 0.008 \mu \mathrm{m}$ (Fig. $2 \mathrm{~B}$ ). Polymer postprocessing procedures such as sanding resulted in a similar surface roughness of $\sim 4 \mu \mathrm{m}$ based on computed arithmetic calculations (Fig. 2C, D). Additionally, QHM polymers exhibited contact angles of less than $90^{\circ}$ for all samples, demonstrating hydrophilicity and suitability for subsequent GF biopatterning, with only the 0 s UV QHM polymer exhibiting a slightly reduced contact angle (by $\sim 10^{\circ}$ ) relative to those of the $90 \mathrm{~s} \mathrm{UV}, 180 \mathrm{~s} \mathrm{UV}$, and $300 \mathrm{~s}$ UV QHM polymers (Fig. 2E, F). Thus, QHM polymer fabrication and postprocessing did not change pore size or surface roughness but had a minor impact on porosity and hydrophilicity.

\section{Effect of polymer fabrication (UV exposure) on mechanically graded QHM polymer material stiffness (microscale elastic modulus)}

To determine if the QHM polymer can mimic the bonetendon interface within physiologically relevant values, i.e., length of the human bone-tendon interface:
$0.1-1 \mathrm{~mm}^{10}$, mechanically graded QHM polymers with 4 different graded regions spanning 1-2 $\mathrm{mm}$ were fabricated. In general, a trend of increased microscale stiffness with increased UV exposure time was observed (Fig. 3 and Fig. S1). Thus, QHM polymers with controlled UV exposure can be fabricated as mechanically graded materials that mimic the physiological dimensions of the bone-tendon interface.

\section{Effect of material stiffness and GFs (nonpatterned soluble and biopatterned) on osteoblast and tenocyte differentiation in vitro}

To determine the combinatorial effect of QHM material stiffness (as a macroscale biomechanical cue) and GFs (as biochemical cues) on bone and tendon cell differentiation, $\mathrm{C} 2 \mathrm{C} 12$ cells, which are a myoblast cell line capable of differentiating into osteoblasts and tenocytes ${ }^{16,17}$, were cultured in the presence of bone- and tendon-promoting GFs on various QHM polymers. Different UV exposure times $(0,90,180$, and $300 \mathrm{~s})$ resulted in QHM polymers with macroscale tensile modulus values of $0.6,1.7,2.5$, and $2.7 \mathrm{GPa}$, respectively, as described in our prior work ${ }^{9}$ and here (Fig. S2). Such mechanical properties approximate the tensile modulus of the human supraspinatus tendon $(0.2-0.6 \mathrm{GPa})^{1}$ and cortical bone $(11.0-29.0 \mathrm{GPa})^{14}$.

Initially, soluble (i.e., nonpatterned) GFs were added to the culture medium to ensure that the cells in different QHM polymer groups received similar dosages, thus eliminating any potential confounding variables as a result of differential GF binding to patterned substrates. ALP staining and von Kossa staining were used to test osteogenic differentiation, and IF staining (for the markers SCX, TNMD, and TNC) was used to test tenogenic differentiation. BMP-2 was used as a bone-promoting GF cue based on previous studies ${ }^{15-17}$. When cultured in the presence of BMP-2 for 4 and 6 days, C2C12 cells exhibited increased activity of the osteoblast marker ALP on stiffer QHM polymers (Fig. 4A). With further culture (27 days), comparable $\mathrm{C} 2 \mathrm{C} 12$ mineralization, as indicated by von Kossa staining, was observed on QHM polymers in all groups (Fig. 4B). It is worth noting that BMP-2 can also induce chondrogenesis with increased ALP activity ${ }^{21}$, which is not discussed here. Thus, QHM biomaterial macroscale stiffness has an early effect on BMP-2mediated $\mathrm{C} 2 \mathrm{C} 12$ osteoblast-like differentiation.

Material stiffness also affected $\mathrm{C} 2 \mathrm{C} 12$ tenocyte differentiation. FGF-2 was used as a tendon-promoting GF cue based on previous studies ${ }^{16,17}$. In our study, FGF-2 increased the expression of TNC and TNMD of C2C12 cells in a dose-dependent manner when the cells were cultured on TCPS for 3 days, and $100 \mathrm{ng} / \mathrm{mL}$ FGF-2 showed increased $\mathrm{C} 2 \mathrm{C} 12$ tenogenic differentiation relative to that of the $0 \mathrm{ng} / \mathrm{mL}$ and $50 \mathrm{ng} / \mathrm{mL}$ FGF-2 groups. When cultured in the presence of FGF-2 for 3 days, C2C12 cells 

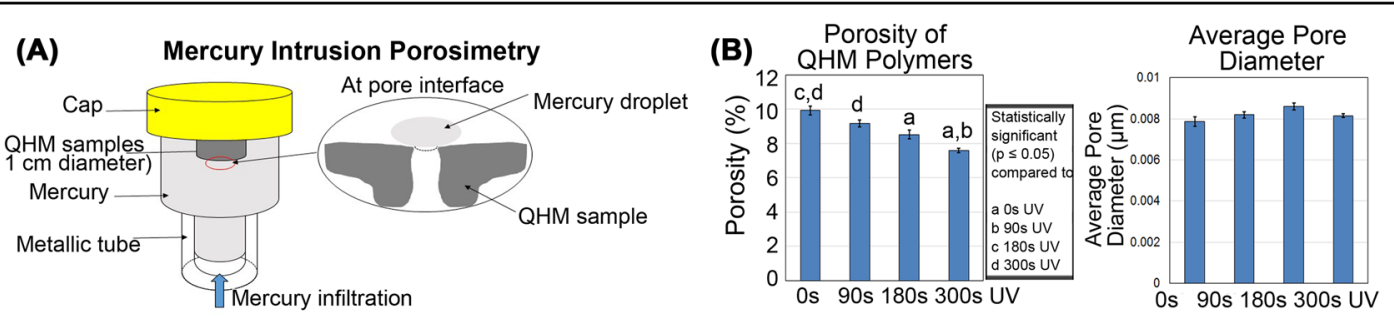

(C)

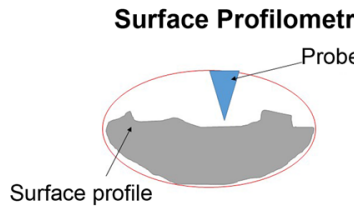

(E)

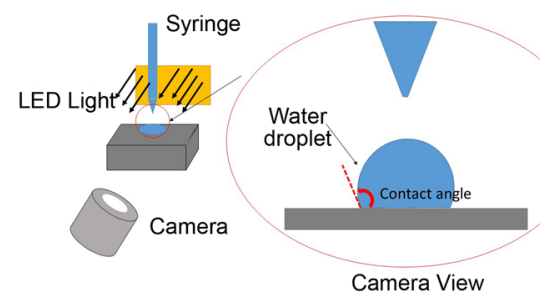

(F)

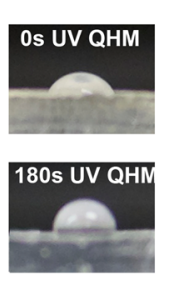

(D) Surface Roughness of

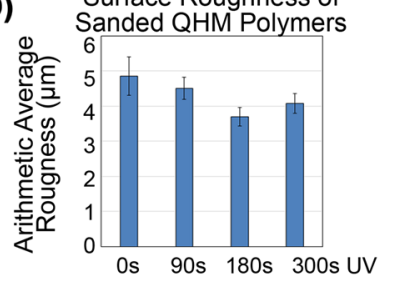

Fig. 2 Effect of UV exposure and sanding on (nongraded) QHM polymer porosity, roughness, and hydrophobicity (contact angle). A Schematic diagram depicting mercury intrusion porosimetry. B Average porosity and pore size of QHM polymers. Porosity decreased by less than $2.5 \%$ with increasing UV exposure $(n=3)$. Average pore diameter of QHM polymers. Pore size was not altered by UV exposure $(n=3)$. C Schematic diagram depicting line profilometry. D Surface roughness of QHM polymers. The computed arithmetic average roughness remained unchanged by sanding $(n=3)$. E Schematic diagram depicting hydrophobicity (contact angle) measurements. $\mathbf{F}$ Hydrophobicity (contact angle) of QHM polymers. The contact angle increased by $10^{\circ}$ with increased UV exposure between the $0 \mathrm{~s}$ UV with $90 \mathrm{~s}$ UV QHM polymer and remained unaltered thereafter $(n=6)$. Error bars indicate the standard error of the mean. Statistical significance as indicated.

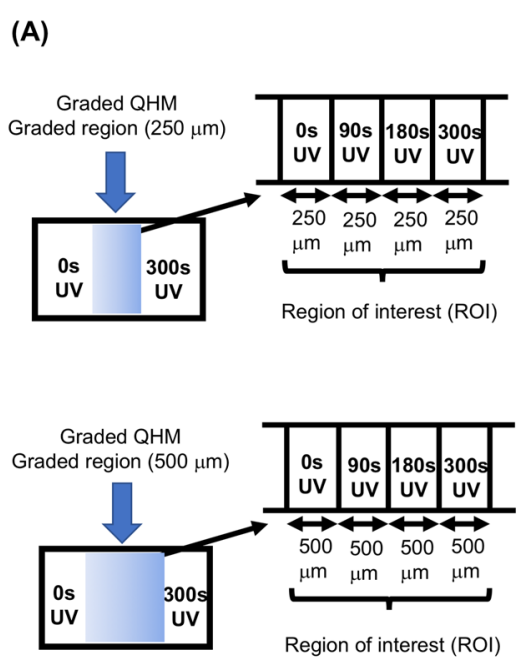

(B) Normalized elastic modulus
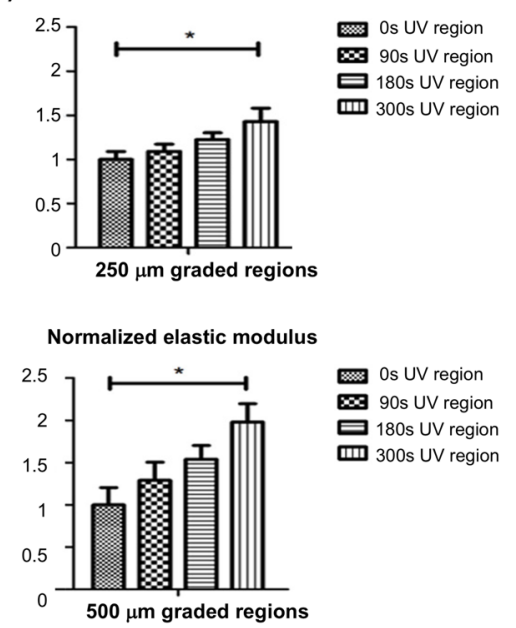

0s UV region
90s UV region
$\square$ 180s UV region 180s UV region

Fig. 3 Effect of UV exposure on the mechanically graded QHM polymer microscale elastic modulus. A Schematic diagram depicting the fabrication of mechanically graded QHM with graded regions of 250 and $500 \mu \mathrm{m}$. B Normalized elastic modulus of mechanically graded QHM. Graded regions spanning $250 \mu \mathrm{m}(n=12)$ and $500 \mu \mathrm{m}(n=6)$ in width were fabricated, and an increasing gradient of elastic moduli was observed with increased UV exposure. Error bars indicate the standard error of the mean; statistical significance. ${ }^{*} p \leq 0.05$. 


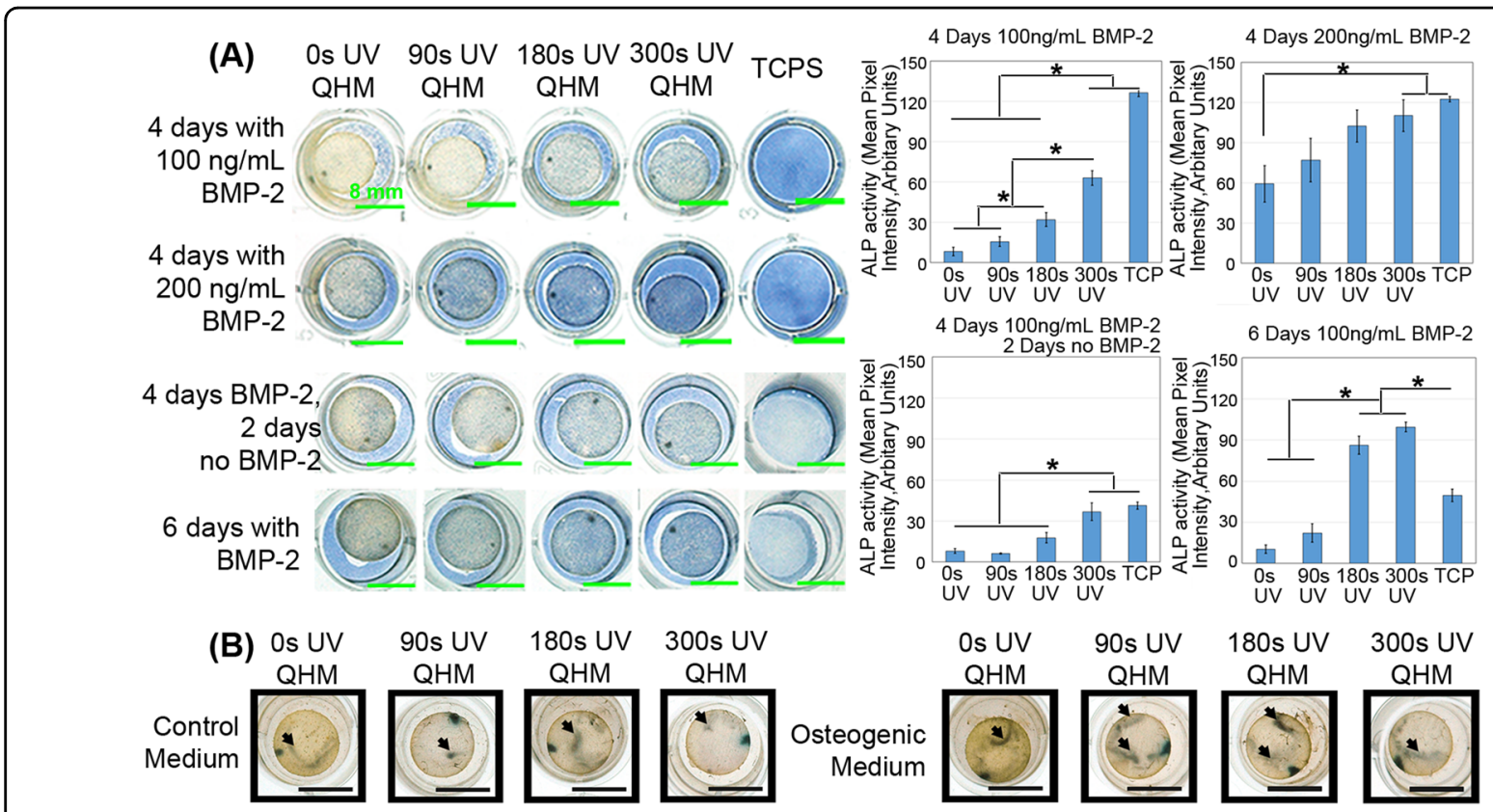

Fig. 4 Effect of (nongraded) QHM polymer stiffness on C2C12 osteoblast differentiation in the presence of soluble GF cues (BMP-2). C2C12 cells were cultured on QHM polymers with different material stiffnesses (as a result of different UV exposure) at designated time points. A After 4-6 days of culture in either soluble $100 \mathrm{ng} / \mathrm{mL}$ or $200 \mathrm{ng} / \mathrm{mL} \mathrm{BMP-2-containing} \mathrm{medium,} \mathrm{increased} \mathrm{ALP} \mathrm{staining} \mathrm{of} \mathrm{C2C12} \mathrm{cells} \mathrm{was} \mathrm{observed} \mathrm{on}$ QHM polymers with increased substrate stiffness. This trend was impacted by the duration (4 days BMP-2 +2 days no BMP-2 versus 6 days BMP-2) of BMP-2 administration $(n=3)$. B After 27 days of culture, comparable C2C12 cell-mediated mineralization indicated by von Kossa staining was observed on QHM polymers in all groups ( $n=6-9$ ). ALP-positive regions are stained blue. von Kossa-positive regions are stained black (black arrows). Scale bars are as indicated. TCPS, tissue culture-grade polystyrene. Error bars indicate the standard error of the mean; statistical significance. ${ }^{*} p \leq 0.05$.

exhibited decreased expression of the tendon marker Scleraxis (SCX) on stiffer QHM polymers (Fig. 5). Thus, QHM biomaterial macroscale stiffness has an early effect on FGF-2-mediated C2C12 tenocyte-like differentiation.

Our data (Figs. 4 and 5) showed that soluble or liquidphase GFs and biomechanical cues interact. However, some tissue engineering strategies utilize GF-containing scaffolds, where biochemical cues may be conjugated or immobilized in a solid-phase form to facilitate persistent signaling. As such, we aimed to study whether a similar phenomenon occurred with solid-phase biochemical cues interacting with biomechanical cues. Prior to such studies, we first determined GF retention on the scaffolds after inkjet biopatterning ${ }^{15-18,20,21}$. QHM polymers patterned with either BMP-2 or FGF-2 retained pattern fidelity after multiple PBS washes. Although GF desorption was observed following the first PBS wash, no further losses occurred with additional washes (Fig. 6A, B). This demonstrated the persistence of biopatterned GFs, which is crucial for longterm spatial control of musculoskeletal differentiation and has been demonstrated in our prior in vivo studies ${ }^{15,21}$. Furthermore, shelf-life studies demonstrated that GF bioactivity was retained under extended storage conditions at $4{ }^{\circ} \mathrm{C}$, with the majority of GF-biopatterned QHM polymers retaining their ability to spatially direct osteoblast differentiation 3 months post-printing (Fig. S3).
The effect of GF biopatterning and QHM polymer material stiffness on bone and tendon cell differentiation was also examined. BMP-2 and FGF-2 biopatterning on fibrin-coated QHM increased C2C12 ALP staining and SCX expression relative to that of their respective offpattern controls. Additionally, similar to soluble GF studies, GF patterns increased ALP activity and decreased SCX expression in $\mathrm{C} 2 \mathrm{C} 12$ cells with increased material stiffness. The interaction effects were significant for different material stiffnesses and biopatterned BMP-2 on ALP activity $\left(F(3,40)=3.127, p=0.036\right.$, partial $\eta^{2}=$ $0.190)$, as well as for different material stiffness and biopatterned FGF-2 on SCX expression $(F(3,40)=6.428, p=$ 0.001 , partial $\eta^{2}=0.325$; Fig. 7). A similar trend was also observed in C3H10T1/2 cells-increased ALP staining was found on stiffer materials within BMP-2 biopatterned regions (Fig. S4).

Altogether, these data showed that increased QHM polymer stiffness as a result of longer UV exposure (i.e., macroscale tensile moduli: $0.6 \mathrm{GPa}-2.7 \mathrm{GPa}$ based on 0-300 s UV exposure time) ${ }^{9}$, increased BMP-2-mediated $\mathrm{C} 2 \mathrm{C} 12$ osteoblast differentiation and decreased FGF-2mediated tenocyte differentiation, regardless of whether the GF cue was soluble or biopatterned. Thus, GF(s) and material stiffness can be used together to spatially control musculoskeletal cell differentiation in vitro. 


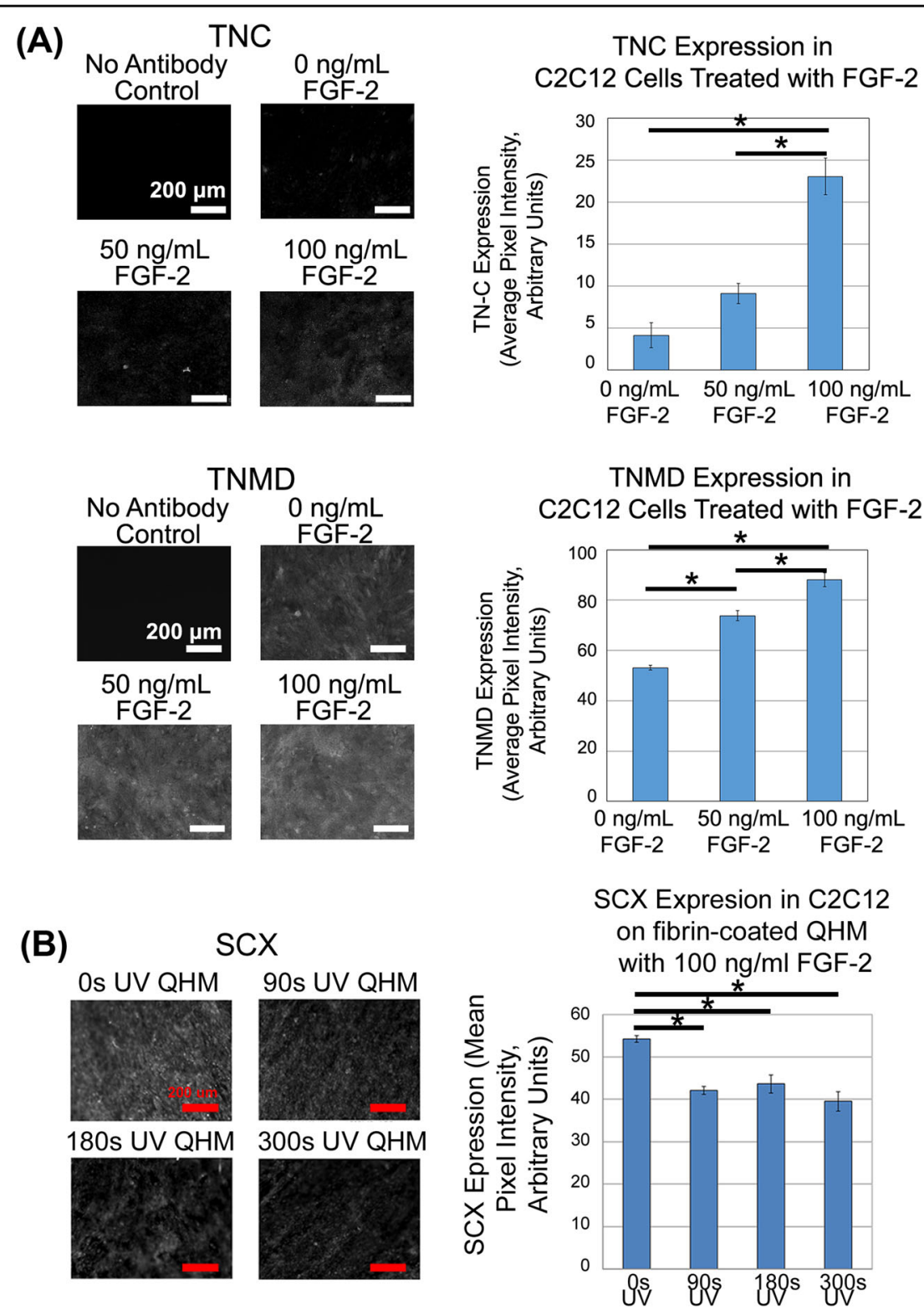

Fig. 5 Effect of (nongraded) QHM polymer stiffness on C2C12 tenocyte differentiation in the presence of soluble GF cues (FGF-2). A FGF-2 increased the expression of TNC and TNMD in C2C12 cells in a dose-dependent manner when the cells were cultured on tissue culture-grade polystyrene. B C2C12 cells were cultured on QHM polymers with different material stiffnesses (as a result of different UV exposure). After 3 days of culture in $100 \mathrm{ng} / \mathrm{mL}$ FGF-2-containing medium, decreased SCX expression was observed with increased substrate stiffness $(n=9)$. TNC-, TNMD-, or SCX-positive regions are shown in white; scale bars are as indicated; error bars indicate the standard error of the mean; statistical significance. ${ }^{*} p \leq 0.05$.

Effect of blebbistatin (a mechanosensing inhibitor) on material stiffness- and GF-mediated osteoblast differentiation

To further determine the involvement of the material stiffness of the QHM polymer on $\mathrm{C} 2 \mathrm{C} 12$ osteoblast differentiation, blebbistatin ${ }^{26}$, which disrupts mechanosensing, was utilized together with soluble GFs in subsequent experiments. As such, C2C12 cells were cultured on QHM polymers with various tensile moduli in the presence of BMP-2, with or without $25 \mu \mathrm{M}$ blebbistatin (Fig. 8A).
Our data showed that BMP-2-induced ALP activity was slightly inhibited by blebbistatin supplementation, although the overall trend of increased ALP activity on stiffer QHM polymers remained $(F(1,20)=98.559, p \leq$ 0.001 , partial $\left.\eta^{2}=0.831\right)$. Indeed, the interaction effect of different material stiffnesses and blebbistatin treatment on BMP-2-induced ALP activity was significant $(F(4,20)=$ 14.768, $p \leq 0.001$, partial $\left.\eta^{2}=0.747\right)$. The level of blebbistatin-induced ALP inhibition was determined by calculating the ratio of ALP activity between the $25 \mu \mathrm{M}$ 
(A) Representative Images of Immobilized to Fibrin-Coated QHM Polymers

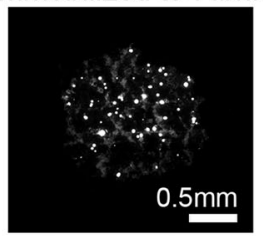

Before wash (0.5 $\mu \mathrm{L}$ Handprinted)

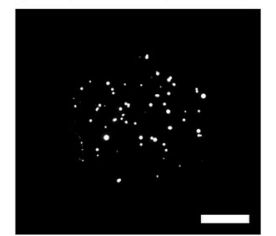

2nd PBS wash (5 min)

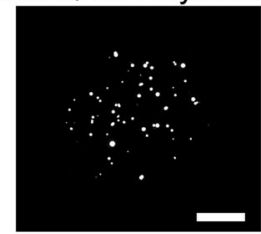

1st PBS wash (5 min)

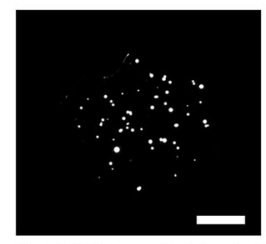

3rd PBS wash (5 min)

Representative Images of

(B) Dylight 650-Labeled FGF-2

Immobilized to Fibrin-Coated QHM Polymers

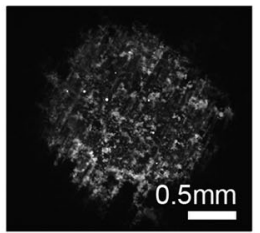

Before wash (0.5 $\mu \mathrm{L}$ Handprinted)

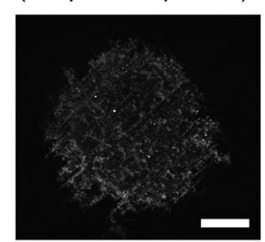

2nd PBS wash (5 min)

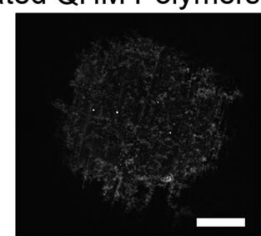

1st PBS wash (5 min)

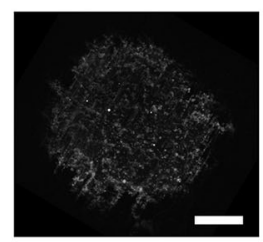

3rd PBS wash (5 min)
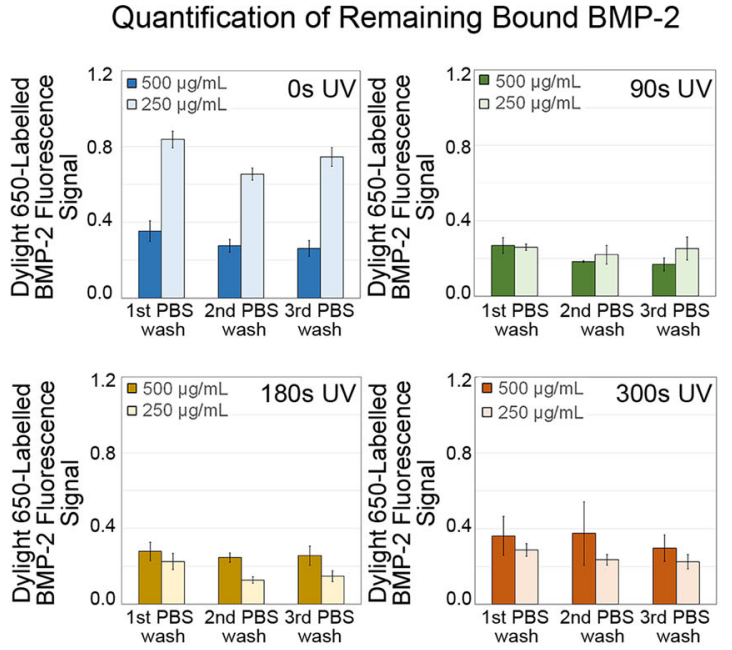

Quantification of Remaining Bound FGF-2
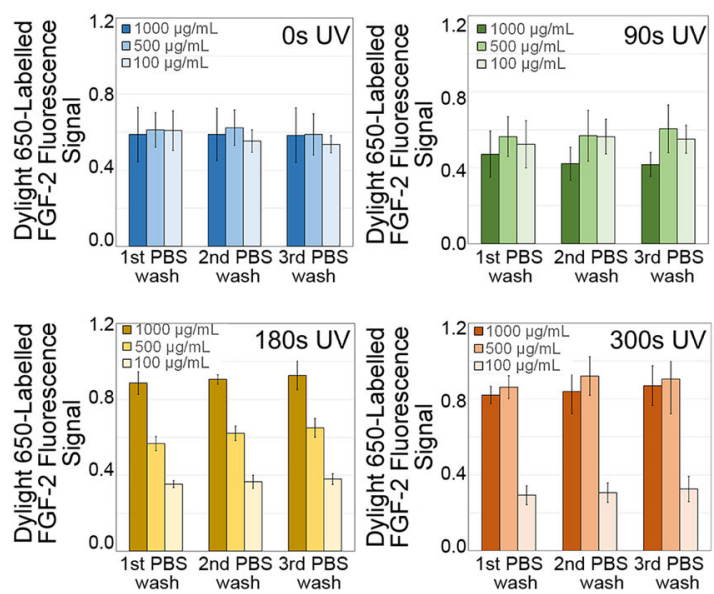

Fig. 6 Effect of fibrin coating on (nongraded) QHM polymer immobilization of GFs. A BMP-2 immobilization on fibrin-coated QHM polymers. B FGF-2 immobilization on fibrin-coated QHM polymers. Successive washing did not alter the amount of fluorescently labeled BMP-2 or FGF-2, demonstrating persistent immobilization $(n=3)$. Fluorescence intensity was normalized to sample images acquired prior to washing; error bars indicate the standard error of the mean.

blebbistatin-treated group and its corresponding $0.3 \%$ DMSO vehicle control (ALP inhibitory index values of 0.0 and 1.0 indicate complete inhibition and no inhibition, respectively). Analysis indicated that a higher level of C2C12 ALP inhibition was observed on less stiff $0 \mathrm{~s}$ UV QHM polymers, e.g., C2C12 ALP inhibition in $0 \mathrm{~s} \mathrm{UV}$ QHM was higher than that in 300 s UV QHM, demonstrating that cells on less stiff materials were more sensitive to mechanosensing than cells on stiffer materials. With increased culture duration, inhibition of ALP activity was observed only on the least stiff QHM polymer ( $0 \mathrm{~s}$ UV) at 8 days and abrogated by 14 days (Fig. $8 \mathrm{~B}$ ).
These results suggested that material stiffness had an obvious effect on the early but not the late phase of BMP2-mediated osteoblast differentiation. The eventual osteogenic differentiation and mineralization of cells on all QHM polymers may be explained by the prolonged stimulatory effects of BMP-2, which eventually overcomes material stiffness effects, as an increased frequency of BMP-2 administration or an increased dose also increased ALP activity (Fig. 4A). Thus, the varying levels of osteoblast and tenocyte differentiation observed during culture on QHM polymers of varying stiffness in vitro were attributed to mechanosensing effects. 


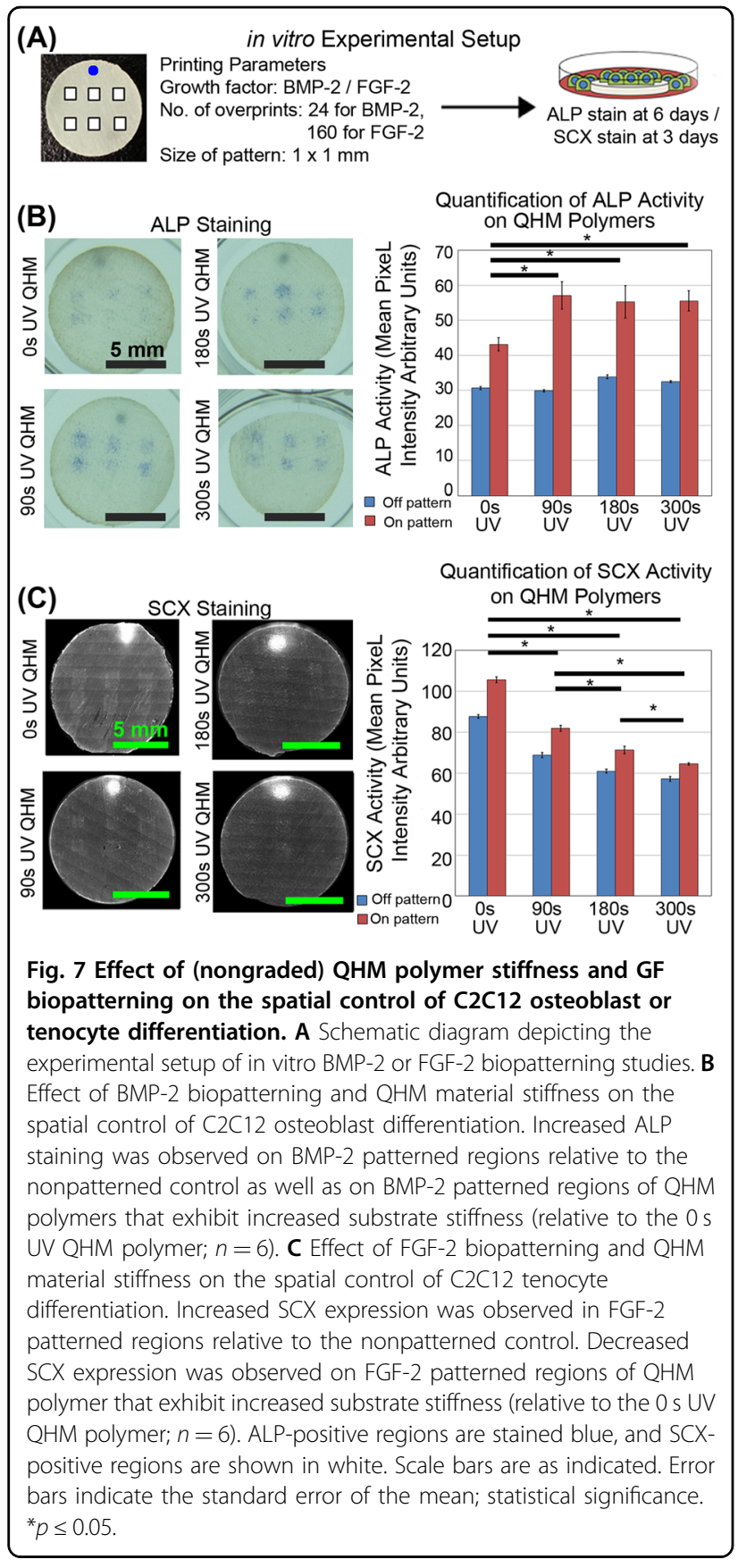

Effect of GF-biopatterned, mechanically graded (biphasic) QHM polymers on musculoskeletal differentiation in vitro

\section{and in an ectopic, mouse subcutaneous implantation model}

Having established that material stiffness and GFs interact to affect musculoskeletal differentiation on uniform, nongraded QHM polymers, we next assessed the combinatorial effect of mechanically graded QHM polymers and GFs on bone and tendon cell differentiation in vivo. To simplify the study design, a biphasic bone- tendon scaffold consisting of only $0 \mathrm{~s}$ UV and $300 \mathrm{~s}$ UV QHM polymer regions was used. Owing to its phototunable properties, this biphasic material approximates the tensile attributes of the human supraspinatus tendon and cortical bone ${ }^{9}$, which is most commonly torn in rotator cuff tears ${ }^{1}$.

Similar to prior in vitro culture conditions, C2C12 cells were cultured on mechanically graded QHM polymers in the presence of liquid-phase bone- or tendon-promoting GFs, i.e., BMP-2 and FGF-2, respectively. Our data showed that the stiffer (300 s UV) region of QHM polymers showed increased ALP staining but decreased SCX expression relative to less stiff ( $0 \mathrm{~s} \mathrm{UV})$ regions and vice versa (Fig. S5), which was similar to the results for nongraded QHM polymers.

Subcutaneous implantation of biphasic, fibrin-coated QHM polymers patterned with bone- and tendonpromoting cues in mice demonstrated biocompatibility, as well as the formation of bone- and tendon-like tissues relative to nonprinted controls. After implantation, the mice showed consistent weight gain and did not exhibit adverse clinical signs or mortality, including detrimental reactions such as necrosis, infection, and granuloma around the implants (data not shown). Scaffold regions biopatterned with BMP-2 (300s UV QHM) resulted in bone-like tissue with the formation of collagen-rich structures (shown in trichrome and hematoxylin \& eosin/H\&E staining) that contained abundant bone marrow and bone cells, including multinucleated, tartrateresistant acid phosphatase/TRAP-positive osteoclasts, as well as cuboid-shaped osteoblasts that lined the bone surface. Scaffold regions biopatterned with FGF-2 (0 s UV QHM polymer) resulted in tendon-like tissues with birefringent (shown in polarized light microscopy), wavy and crimped collagen fibers (shown in trichrome and $\mathrm{H} \& \mathrm{E}$ staining) that contained cells that strongly expressed the tenocyte marker SCX (Fig. 9 and S6). Altogether, these data demonstrated that bone- and tendon-promoting GF biopatterning along with biphasic scaffolds with boneand tendon-like macroscale biomechanical properties spatially controlled ectopic bone- and tendon-like tissue formation in vivo.

\section{Discussion}

In this study, we built upon our prior work ${ }^{9}$ by utilizing phototunable, mechanically robust QHM polymers as models of functionally graded scaffolds to investigate the interplay between scaffold biomechanical properties and biological augmentation. Specifically, this approach included spatially controlled, bone- and tendon-like material stiffness (macroscale biomechanical cues) as well as biopatterned bone- and tendon-promoting GFs (biochemical cues) to combinatorially mediate multitissue-specific differentiation. 


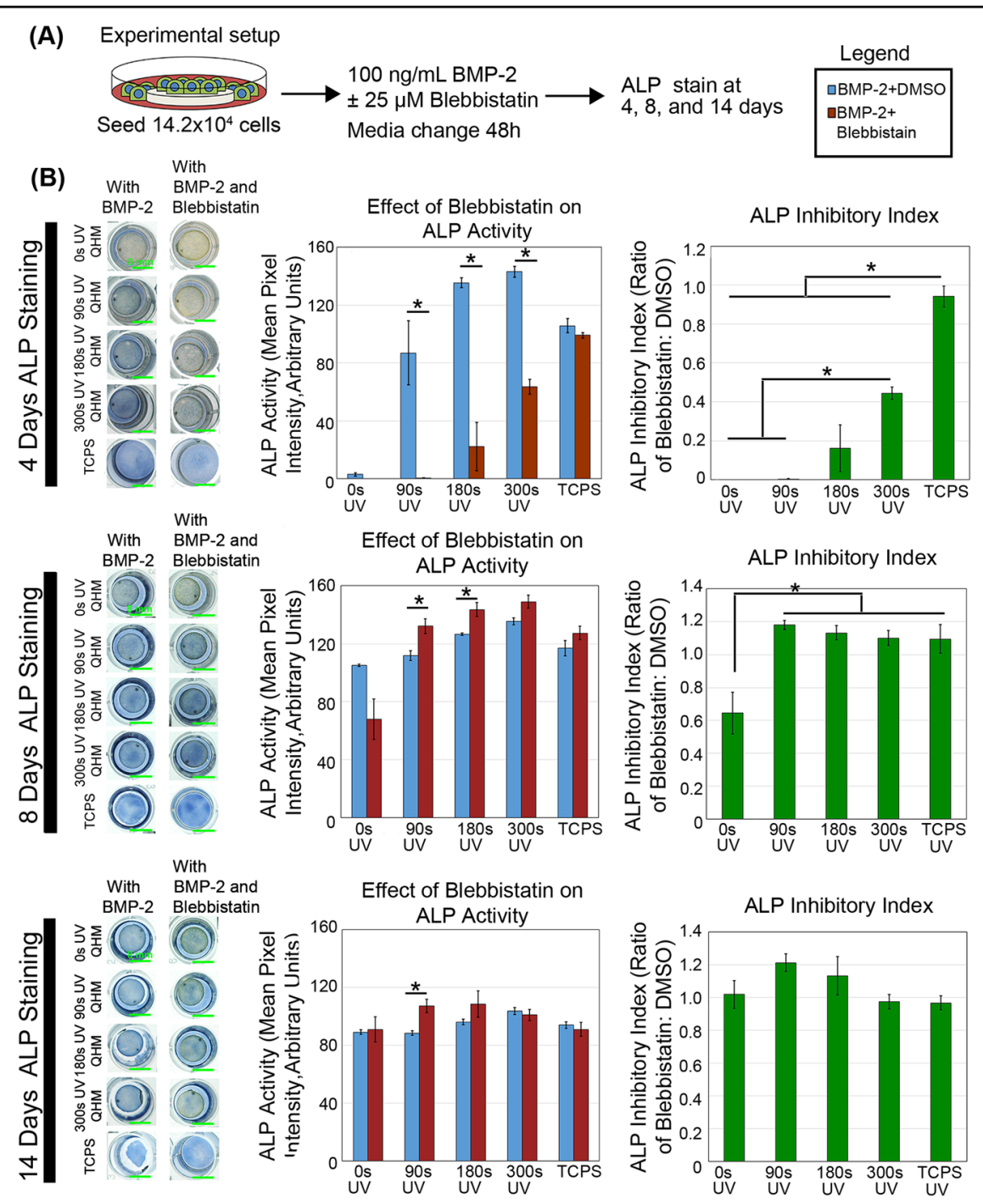

Fig. 8 Effect of (nongraded) QHM polymer stiffness on C2C12 osteoblast differentiation with or without $25 \mu \mathrm{M}$ blebbistatin. A Schematic diagram depicting the experimental setup of in vitro mechanosensing studies. B Effect of QHM polymer stiffness on soluble BMP-2-mediated C2C12 osteoblast differentiation after 4,8 , and 14 days of culture in the presence or absence of $25 \mu \mathrm{M}$ blebbistatin. After 4 days of culture, increased ALP staining was observed with increased substrate stiffness. Blebbistatin inhibited substrate stiffness-mediated ALP staining, but the overall trend of increased ALP staining with increasing substrate stiffness remained. Blebbistatin-mediated inhibitory effects were more obvious on less stiff QHM polymers and less obvious after longer culture periods. The ALP inhibitory index was defined as the ratio of ALP activity for blebbistatin-treated cells to DMSO-treated cells; a value of 1.0 indicated no inhibition, while a value of 0.0 indicated complete inhibition $(n=3)$. ALP-positive regions are stained blue. TCPS, tissue culture-grade polystyrene. Error bars indicate the standard error of the mean; statistical significance. ${ }^{*} p \leq 0.05$.

Successful repair of hard-to-soft multitissue units such as rotator cuff tears can be challenging. To restore shoulder function, contemporary rotator cuff surgeries employ suturing for mechanical reapproximation of bone and tendon tissues and humeral bone decortication to encourage infiltration of bone marrow-derived cells for biologically mediated tissue healing ${ }^{27}$. Current tissue engineering strategies have mirrored clinical approaches, pursuing the development of mechanically robust scaffolds and biological augmentation. From a mechanical standpoint, spatial control of scaffold mechanical properties with multitissue bone- and tendon-like attributes will be necessary to sustain musculoskeletal movement and reduce stress concentrations between mechanically distinct regions to avoid biomaterial failure ${ }^{9}$. From a biological standpoint, spatial control of scaffold bioinductive properties will be vital for multiphenotype musculoskeletal differentiation to biologically augment multitissue healing $9,10,12,28$. Although there have been significant advances in the field of mechanically graded scaffolds (or grafts) ${ }^{3,4,7-9,29,30}$, only a limited number of studies have attained bone- and tendon-like mechanical 


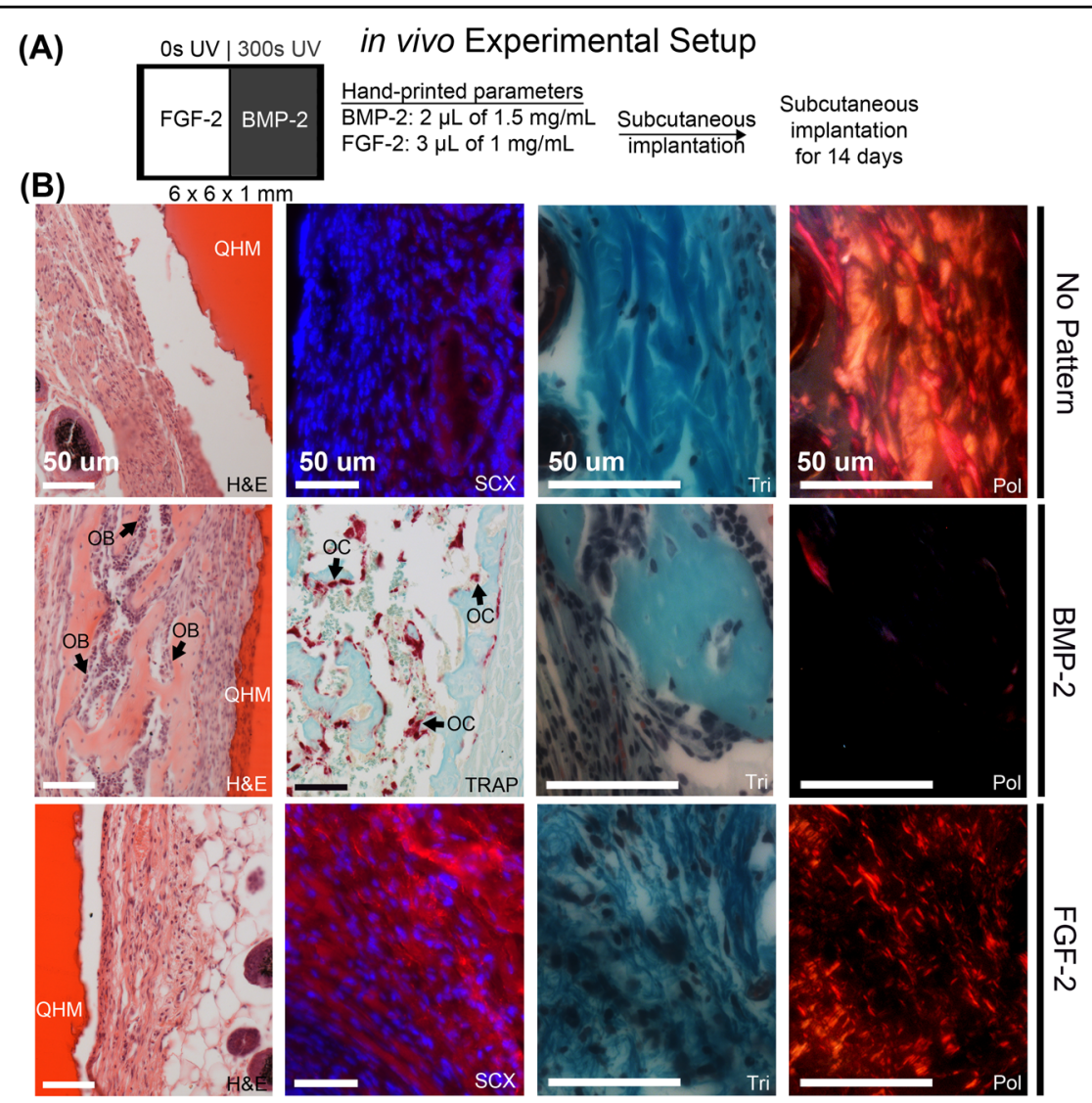

Fig. 9 Effect of mechanically graded QHM polymer stiffness and GF biopatterning on the spatial control of ectopic bone- and tendon-like tissue formation. A Schematic diagram depicting the experimental setup of in vivo BMP-2 and FGF-2 biopatterning studies. B Effect of BMP-2 and FGF-2 biopatterning on the spatial control of bone- and tendon-like differentiation after 14 days of subcutaneous implantation in mice. SCX-positive regions containing birefringent and wavy collagen fibers reminiscent of tendon-like tissue were observed on QHM polymers patterned with FGF-2; TRAP-positive, collagen-rich extracellular structures that contained bone cells and bone-marrow tissue reminiscent of bone-like tissue were observed on QHM polymers patterned with BMP-2 $(n=9)$. Scale bars are as indicated.

properties at the macroscale level, which is vital for translational applications for shoulder function and repair ${ }^{9}$. There have also been reports of graded scaffolds that utilized calcium phosphate gradients to pattern heterotypic cell populations ${ }^{4,6,7}$. An outstanding question remains as to whether multitissue phenotypes, as opposed to gradations in osteoblast phenotype, were patterned since calcium phosphate is typically regarded as an osteogenic $^{31}$ and not a tenogenic ${ }^{11}$ factor. To the best of our knowledge, there are no reports of functionally graded scaffolds with spatially controlled bone-tendon macroscale mechanical properties and bioinductive cues, which are crucial for both mechanical repair and spatially precise restoration of multitissue phenotypes.

Within this context, material stiffness has long been recognized as an important factor for controlling cell differentiation and tissue regeneration. Studies on cellular biomechanics showed that compliant materials promoted neural and myogenic differentiation, whereas stiffer materials promoted osteoblast differentiation ${ }^{13}$. However, it remains unclear whether such mechanosensing effects in various contexts share similar mechanisms. Mechanotransduction pathways are typically activated when integrin-mediated cell-ECM interactions transmit mechanosignals from the environment into the cell nucleus through cytoskeletal components such as actin and initiate/regulate cell behaviors accordingly ${ }^{32,33}$. In addition to cell-ECM adhesion sites, other sites, such as cell-cell junctions and ion channels, have also been implicated in mechanotransduction signals, which are not discussed in detail here. Additionally, human mesenchymal stem cells have even been reported to recall their past culture history on soft or stiff materials, which can influence musculoskeletal differentiation via a "mechanical memory and dosing" effect ${ }^{34}$. While such studies have established that material stiffness influences cell behavior, it is worth noting that prior studies ${ }^{4,13}$ typically utilized microscale approaches such as atomic force microscopy or microindentation to derive mechanical properties such as Young's modulus. While such microscale mechanical 
attributes are biomechanically relevant to cells, macroscale mechanical properties are critical for sustaining physiological loading and maintaining scaffold integrity. This subtle but important distinction must be recognized since the same scaffold can exhibit significantly different moduli at both the microscale and macroscale levels ${ }^{35}$ (Figs. S1 and S2). In this respect, QHM polymers were ideal model substrates for assessing the effect of macroscale mechanical properties on cell behavior since 0,90 , 180, and $300 \mathrm{~s}$ UV exposure attained macroscale tensile moduli of $0.6,1.7,2.5$, and $2.7 \mathrm{GPa}$, respectively ${ }^{9}$, and these values closely approximate the macroscale tensile modulus values of human supraspinatus tendon $(0.2-0.6 \mathrm{GPa})^{1}$ and cortical bone $(11.0-29.0 \mathrm{GPa})^{14}$. Additionally, the photocrosslinkable nature of QHM polymers enabled tunable stiffness gradients with macroscale bone- and tendon-like properties at resolutions $\left(0.25-0.5 \mathrm{~mm}\right.$ using a photomask; our prior study ${ }^{9}$ and Fig. 3) adequate for mimicking the dimensions of bonetendon tissues $(0.1-1 \mathrm{~mm} \text { in length })^{10}$. In this study, we observed a trend of increased microscale stiffness (elastic modulus measured by the microindenter) with increased UV exposure time, and achieved a similar elastic modulus in our graded specimens with a resolution between $0.25 \mathrm{~mm}$ and $0.5 \mathrm{~mm}$ using a photomask. However, a statistically significant difference in the elastic modulus was observed only between the $0 \mathrm{~s}$ and $300 \mathrm{~s}$ UV QHM regions. This may be due to technical limitations in our setup (limited optical performance of our collimating lens) and can be improved with a better collimating lens or use of a collimated light source (e.g., laser) during graded material fabrication.

GF biopatterning methodology is based on printing exogenous GFs onto specified regions of native ECM materials at micrometer-scale resolutions ${ }^{9,15-18,20,21}$, where they absorb into and become immobilized to ECM such as fibrin via native ECM-GF binding interactions ${ }^{20}$. This biomimics how GFs are locally sequestered in nature $^{36}$ and is distinct from traditional drug delivery methods that are dependent on carrier-mediated release and subsequent GF diffusion kinetics ${ }^{37}$. Instead, GFs are immobilized onto a scaffold at low picogram- to nanogram-level doses (our prior work ${ }^{20}$ and Figs. 6, 7, and 9 of this study) to provide persistent GF signaling that spatially directs cell phenotype(s) while also minimizing off-target effects. As a result, spatial control of multiple cell or tissue phenotypes is achieved in vitro ${ }^{16-18}$ and in vivo ${ }^{15,21}$. Thus, GF biopatterning is ideally suited for engineering multitissue units.

In vitro, our data demonstrate that increased material stiffness promoted GF-mediated osteoblasts and compromised GF-mediated tenocyte differentiation (Figs. 4, 5, 7, 8, S3, S4, and S5). Interestingly, long-term or extended GF administration abrogates this effect, indicating the dominance of biochemical cues from growth factors over macroscale biomechanical cues, at least based on this study's experimental settings (Figs. 4 and 8). Additionally, osteoblast and tenocyte differentiation was invariant to material stiffness in regions where no GF was present (see off-pattern controls in Fig. 7B, C; the slight decrease in off-pattern controls for SCX expression may be attributed to polymer autofluorescence). This contrasts with prior studies where material stiffness alone influenced cell differentiation $^{4,13}$, which may be attributed to differences in experimental conditions, including cell types, culture media, and material chemical compositions. Such experimental variations are expected to influence the presence of stretch-activated channels and ultimately affect cell mechanosensitivity ${ }^{38}$. In vivo, an ectopic subcutaneous implantation model was used to demonstrate the clinical potential of GF-biopatterned, mechanically graded scaffolds for bone-tendon repair. Ectopic boneand tendon-like tissues were observed simultaneously within a single biphasic (0 s UV and $300 \mathrm{~s}$ UV QHM polymer) scaffold (Fig. 9), demonstrating de novo multitissue formation.

Although not addressed in this study, several elements are worth considering in future work. (1) Our study utilized a subcutaneous implantation model to primarily assess the potential use of GF-biopatterned, mechanically graded QHM polymer for bone-tendon repair. In our future work, the use of acute rupture or chronic degenerated, large-to-massive tendon injury animal models, such as in rats, rabbits, ovines, canines, bovines and primates, will better mimic clinical conditions ${ }^{39}$. (2) Fibrochondrocytes are present in the mineralized and unmineralized fibrocartilage regions of the bone-tendon interface ${ }^{9,10,12,28}$. However, they were not spatially patterned here as our intent was to reduce material complexity. Transitional regions (i.e., fibrochondral regions) of the mouse shoulder develop and mineralize postnatally, and such processes are impaired when mechanical loading is disrupted ${ }^{10}$. This raises the possibility that fibrochondral regions can be regenerated following implantation of a graded scaffold containing bone- and tendon-like cells and restoration of physiological shoulder loading forces. Therefore, incorporating mechanical conditioning may provide an avenue for fibrochondrocyte patterning without having to resort to additional GFs ${ }^{40}$. (3) To simplify the study design both in our in vitro and in vivo work, we chose to use a biphasic scaffold consisting of only $0 \mathrm{~s} \mathrm{UV}$ and $300 \mathrm{~s}$ UV QHM to mimic the tensile attributes of the human supraspinatus tendon and cortical bone, respectively ${ }^{9}$. If necessary, the effect of multiphasic stiffness or a true stiffness gradient on cell differentiation could be assessed in the future. This can be achieved by simple application of UV light in conjunction with photomasks on QHM polymers at physiologically relevant length 
scales (our prior study ${ }^{9}$ and Fig. 3 of this study). (4) FGF-2 was used as the only tendon-promoting GF in the current study. The combined or synergistic effects of other material biophysical and biological cues can be studied and compared. These biological cues include GFs, hormone-related peptides, and ECM components that have been shown to play a role in enthesis repair ${ }^{41}$, e.g., transforming growth factor (TGF)- $\beta 1$, TGF- $\beta 3$, growth differentiation factor 7 (GDF-7), Indian hedgehog (Ihh), parathyroid hormone-related peptide (PTHrP), insulinlike growth factor-I (IGF-I), and extracted bone/cartilage $\mathrm{ECM}^{42}$. For biophysical cues, these also include material geometric cues/topography, porosity/pore size, and biomechanical loading ${ }^{43}$. Such studies will be useful to enhance lineage-specific cell differentiation and tissue regeneration as a means to further improve musculoskeletal integration of these scaffolds.

\section{Conclusion}

In summary, we have developed a novel strategy for the spatial control of multiple musculoskeletal tissue phenotypes. This was achieved by combining bone- and tendonlike material stiffness (as macroscale biomechanical cues) with multitissue-specific GF biopatterning (as biochemical cues), which can potentially be used for a model system and/or as a graft for hard-to-soft, multitissue repair. This versatile strategy can also be broadly applied to pattern multitissue units and holds promise for tissue engineering and regenerative medicine strategies for the repair of multitissue injuries.

\begin{abstract}
Acknowledgements
D.W. and D.F.E.K. equally contributed to the work. This work was supported by funding from the AO Foundation Startup Grant (S13-134K, D.F.E.K.), The Chinese University of Hong Kong (D.F.E.K. and D.W.), the Innovation and Technology Fund of Hong Kong (No. ITS/333/18, D.W.; No. ITS/090/18, D.F.E.K.), the Stanford Spectrum MedTech Pilot Award (D.F.E.K., E.C.), the Department of Defense (W81XWH-20-1-0343; Y.P.Y.), NIH grants R01AR057837 (Y.P.Y.), R01AR074458 (Y.P.Y.) and U01AR069395 (Y.P.Y.), the Stanford Biomedical Mini Seed Grant (Y.P.Y.), the Stanford Coulter Seed Grant (Y.P.Y.) and the Boswell Foundation (Y.P.Y.). We would like to thank S.G. Yerneni (Carnegie Mellon University) for assistance with GF printing. We would like to thank J. Tok and the Soft and Hybrid Materials Facility (Stanford University) for assistance with and access to profilometer instruments. We would like to thank T. Doyle and the $\mathrm{SCl}^{3}$ Small Animal Imaging Service Center (Stanford University) for assistance with and access to microCT instruments. We would like to thank $C$. Au, K.M. Chan, A. Cheung, J. Lai, and S. Wong for assistance with and access to Histology as well as Microscope and Imaging Core Facilities (The Chinese University of Hong Kong). We would like to thank I. Cheng (Stanford University), J. Helms (Stanford University), D. Wan (Stanford University), and D. H.K. Chow (Department of Orthopedics and Traumatology, The Chinese University of Hong Kong) for generous use of their equipment and facilities.
\end{abstract}

\footnotetext{
Author details

${ }^{1}$ Department of Orthopaedic Surgery, Stanford University, 240 Pasteur Drive, Stanford, CA 94304, USA. ${ }^{2}$ Institute for Tissue Engineering and Regenerative Medicine, The Chinese University of Hong Kong, Shatin, Hong Kong SAR. ${ }^{3}$ School of Biomedical Sciences, Faculty of Medicine, The Chinese University of Hong Kong, Shatin, Hong Kong SAR. ${ }^{4}$ Key Laboratory for Regenerative Medicine, Ministry of Education, School of Biomedical Sciences, Faculty of Medicine, The Chinese University of Hong Kong, Shatin, Hong Kong SAR.
}

${ }^{5}$ Department of Orthopaedics and Traumatology, Prince of Wales Hospital, Faculty of Medicine, The Chinese University of Hong Kong, Shatin, Hong Kong SAR. ${ }^{6}$ Department of Biological Sciences, University of Pittsburgh, 4249 Fifth Avenue, Pittsburgh, PA 15260, USA. ${ }^{7}$ Engineering Research Accelerator, Carnegie Mellon University, 5000 Forbes Avenue, Pittsburgh, PA 15213, USA. ${ }^{8}$ Department of Biomedical Engineering, Carnegie Mellon University, 5000 Forbes Avenue, Pittsburgh, PA 15213, USA. ${ }^{9}$ Robotics Institute, Carnegie Mellon University, 5000 Forbes Avenue, Pittsburgh, PA 15213, USA. ${ }^{10}$ Department of Material Science and Engineering, Stanford University, 496 Lomita Mall, Stanford, CA 94305, USA. "'Department of Bioengineering, Stanford University, 443 Via Ortega, Stanford, CA 94305, USA

\section{Data availability}

Raw data are available from the corresponding authors upon reasonable request.

\section{Conflict of interest}

The authors declare no competing interests.

\section{Publisher's note}

Springer Nature remains neutral with regard to jurisdictional claims in published maps and institutional affiliations.

Supplementary information The online version contains supplementary material available at https://doi.org/10.1038/s41427-021-00294-z.

Received: 31 July 2020 Revised: 15 December 2020 Accepted: 13 January 2021.

Published online: 12 March 2021

\section{References}

1. Itoi, E. et al. Tensile properties of the supraspinatus tendon. J. Orthop. Res. 13, 578-584 (1995)

2. Rosenbaum, A. J. et al. Histologic stages of healing correlate with restoration of tensile strength in a model of experimental tendon repair. HSS J. 6, 164-170 (2010).

3. Spalazzi, J. P. et al. In vivo evaluation of a multiphased scaffold designed for orthopaedic interface tissue engineering and soft tissue-to-bone integration. J. Biomed. Mater. Res. A 86, 1-12 (2008).

4. Zhu, C., Pongkitwitoon, S., Qiu, J., Thomopoulos, S. \& Xia, Y. Design and fabrication of a hierarchically structured scaffold for tendon-to-bone repair. Adv. Mater. 30, e1707306 (2018).

5. Mozdzen, L. C., Vucetic, A. \& Harley, B. A. C. Modifying the strength and strain concentration profile within collagen scaffolds using customizable arrays of poly-lactic acid fibers. J. Mech. Behav. Biomed. Mater. 66, 28-36 (2017).

6. Lausch, A. J., Chong, L. C., Uludag, H. \& Sone, E. D. Multiphasic collagen scaffolds for engineered tissue interfaces. Adv. Funct. Mater. 28, 1804730 (2018).

7. Li, X. et al. Nanofiber scaffolds with gradations in mineral content for mimicking the tendon-to-bone insertion site. Nano Lett. 9, 2763-2768 (2009).

8. Phillips, J. E., Burns, K. L., Le Doux, J. M., Guldberg, R. E. \& Garcia, A. J. Engineering graded tissue interfaces. Proc. Natl Acad. Sci. USA 105, 12170-12175 (2008).

9. Ker, D. F. E. et al. Functionally graded, bone- and tendon-like polyurethane for rotator cuff repair. Adv. Funct. Mater. 28, 1707107 (2018).

10. Lu, H. H. \& Thomopoulos, S. Functional attachment of soft tissues to bone: development, healing, and tissue engineering. Annu. Rev. Biomed. Eng. 15, 201-226 (2013).

11. Rodeo, S. A. Biologic augmentation of rotator cuff tendon repair. J. Shoulder Elb. Surg. 16, S191-S197 (2007)

12. Yang, P. J. \& Temenoff, J. S. Engineering orthopedic tissue interfaces. Tissue Eng. Part B Rev. 15, 127-141 (2009).

13. Engler, A. J., Sen, S., Sweeney, H. L. \& Discher, D. E. Matrix elasticity directs stem cell lineage specification. Cell 126, 677-689 (2006).

14. Yuehuei, H. A. \& Robert A. D. Mechanical Testing of Bone and the Bone-Implant Interface (CRC Press, 1999).

15. Cooper, G. M. et al. Inkjet-based biopatterning of bone morphogenetic protein-2 to spatially control calvarial bone formation. Tissue Eng. Part A 16, 1749-1759 (2010). 
16. Ker, E. D. et al. Engineering spatial control of multiple differentiation fates within a stem cell population. Biomaterials 32, 3413-3422 (2011).

17. Ker, E. D. et al. Bioprinting of growth factors onto aligned sub-micron fibrous scaffolds for simultaneous control of cell differentiation and alignment. Biomaterials 32, 8097-8107 (2011)

18. Phillippi, J. A. et al. Microenvironments engineered by inkjet bioprinting spatially direct adult stem cells toward muscle- and bone-like subpopulations. Stem Cells 26, 127-134 (2008).

19. Shanjani, Y., Siebert, S. M., Ker, D. F. E., Mercado-Pagán, A. E. \& Yang, Y. P. Acoustic patterning of growth factor for three-dimensional tissue engineering. Tissue Eng. Part A 26, 602-612 (2020).

20. Campbell, P. G., Miller, E. D., Fisher, G. W., Walker, L. M. \& Weiss, L. E. Engineered spatial patterns of FGF-2 immobilized on fibrin direct cell organization. Biomaterials 26, 6762-6770 (2005).

21. Smith, D. M. et al. Precise control of osteogenesis for craniofacial defect repair: the role of direct osteoprogenitor contact in BMP-2-based bioprinting. Ann. Plast. Surg. 69, 485-488 (2012).

22. Tomarken, A. J. \& Serlin, R. C. Comparison of ANOVA alternatives under variance heterogeneity and specific noncentrality structures. Psychol. Bull. 99 90-99 (1986).

23. Krieghoff, J. et al. Increased pore size of scaffolds improves coating efficiency with sulfated hyaluronan and mineralization capacity of osteoblasts. Biomater. Res. 23, 26 (2019).

24. Di Luca, A. et al. Gradients in pore size enhance the osteogenic differentiation of human mesenchymal stromal cells in three-dimensional scaffolds. Sci. Rep. 6, 22898 (2016).

25. Faia-Torres, A. B. et al. Osteogenic differentiation of human mesenchymal stem cells in the absence of osteogenic supplements: a surface-roughness gradient study. Acta Biomater. 28, 64-75 (2015).

26. Kim, D.-H. et al. Actin cap associated focal adhesions and their distinct role in cellular mechanosensing. Sci. Rep. 2, 555 (2012).

27. Gartsman, G. M. Shoulder Arthroscopy (2nd edn). (W.B. Saunders, 2009).

28. Andarawis-Puri, N., Flatow, E. L. \& Soslowsky, L. J. Tendon basic science: Development, repair, regeneration, and healing. J. Orthop. Res. 33, 780-784 (2015).
29. Smith, M. J. et al. Comparison of a novel bone-tendon allograft with a human dermis-derived patch for repair of chronic large rotator cuff tears using a canine model. Arthroscopy 28, 169-177 (2012).

30. Xie, J., Ma, B., Michael, P. L. \& Shuler, F. D. Fabrication of nanofiber scaffolds with gradations in fiber organization and their potential applications. Macromol. Biosci. 12, 1336-1341 (2012)

31. Surmenev, R. A., Surmeneva, M. A. \& Ivanova, A. A. Significance of calcium phosphate coatings for the enhancement of new bone osteogenesis-a review. Acta Biomater. 10, 557-579 (2014)

32. Sun, Z., Guo, S. S. \& Fässler, R. Integrin-mediated mechanotransduction. J. Cell Biol. 215, 445-456 (2016).

33. Chen, C. S. Mechanotransduction - a field pulling together? J. Cell Sci. 121 3285-3292 (2008)

34. Yang, C., Tibbitt, M. W., Basta, L. \& Anseth, K. S. Mechanical memory and dosing influence stem cell fate. Nat. Mater. 13, 645-652 (2014).

35. Smith, R. D. J. et al. Characterizing the macro and micro mechanical properties of scaffolds for rotator cuff repair. J. Shoulder Elbow. Surg. 26, 2038-2046 (2017).

36. Taipale, J. \& Keski-Oja, J. Growth factors in the extracellular matrix. FASEB J. 11, 51-59 (1997).

37. Lin, C. C. \& Anseth, K. S. PEG hydrogels for the controlled release of biomolecules in regenerative medicine. Pharm. Res. 26, 631-643 (2009).

38. Kamkin, A. \& Kiseleva, I. in Mechanosensitivity in Cells and Tissues (eds Kamkin, A \& Kiseleva, I.) (Academia, 2005)

39. Hast, M. W., Zuskov, A. \& Soslowsky, L. J. The role of animal models in tendon research. Bone Jt. Res. 3, 193-202 (2014).

40. Thomopoulos, S. et al. Fibrocartilage tissue engineering: the role of the stress environment on cell morphology and matrix expression. Tissue Eng. Pt A 17, 1039-1053 (2010).

41. Molloy, T., Wang, Y. \& Murrell, G. The roles of growth factors in tendon and ligament healing. Sports Med. 33, 381-394 (2003).

42. Rothrauff, B. B., Yang, G. \& Tuan, R. S. Tissue-specific bioactivity of soluble tendon-derived and cartilage-derived extracellular matrices on adult mesenchymal stem cells. Stem Cell Res. Ther. 8, 133 (2017).

43. Zhang, J. \& Wang, J. H. Mechanobiological response of tendon stem cells: implications of tendon homeostasis and pathogenesis of tendinopathy. J. Orthop. Res. 28, 639-643 (2010). 